\title{
Temporal changes in stress state imaged through seismic tomography
}

\author{
AM Baig ESG Solutions, Canada \\ K Bosman ESG Solutions, Canada \\ TI Urbancic ESG Solutions, Canada
}

\begin{abstract}
In order to understand how the rock mass evolves with extraction in the particularly highly stressed environment of a sill pillar in a hard rock mine in North America, we investigate the utility of seismic tomography as an interpretive tool. We use a rich dataset of blasts to highlight the compressional wave variations in the rock. To understand the optimal spatial resolution, we use checkerboard tests with our input ray path distributions. Although there are areas within the sill pillar that are prone to smearing, we are able to resolve subtle variations in velocity structure. By considering different time periods, we image temporal changes in seismic velocity that we relate to the stress state and damage in the rock. Specifically, we consider data recorded during three sequential time intervals, each consisting of three months of data, associated with the excavation of a stope during the start of the second interval. We observe a high-velocity anomaly in the first time interval that is located to the edge of the future stope. After mining, the high-velocity regions migrate to the other side of the stope and then disperse to the edges of the resolvable area. Equating high-velocity anomalies with high stress gives us the ability to characterise the evolving stress state in the mine and potentially an approach that can be used to avoid hazardous situations.
\end{abstract}

Keywords: sill pillar, high stress mining, seismic tomography

\section{Introduction}

Underground excavations in mines entail a detailed understanding of the stress state to mitigate the risk of rockbursts and other seismicity that may cause damage to infrastructure and potentially casualties to personnel. Depending on the overall mining methodology, the highly stressed areas become more focused in sill and crown pillars. As a mine reaches the end of its lifecycle, the need to safely excavate these areas becomes critical, and techniques that constrain the stress state are essential to enable the safe extraction of the resource.

The rock mass responds to variations in the stress state of the mine; high-stress areas have the effect of closing microcracks in the rock mass and, thereby, increasing seismic velocities. By mapping the velocity variations as a function of space and time we can start to understand the stress variations associated with extraction. Tomographic imaging of mines has been used to relate to the stress distribution in mines in several studies (e.g. Young \& Maxwell 1992; Maxwell \& Young 1996; Silver et al. 2007; Ma et al. 2016). A number of studies have shown promise in using these techniques to monitor the stability of block caving operations (Westman et al. 2012; Mercier et al. 2015).

In this study, we examine data from a North American hard rock mine, where a sill pillar is being extracted utilising a stope and pillar approach. We use blast and seismic events themselves to measure compressional wave travel-times across a seismic monitoring network and reconstruct P-wave velocity images over three consecutive three-month periods: before the extraction of the stope; during extraction; and after extraction. To understand the limitations in the ray geometry, such that the observed velocity anomalies may be confidently interpreted in terms of stress variations, and identify the sensitivity of the observations to picking errors, we employ checkerboard resolution tests. 


\section{Dataset}

In this example, monitoring of seismicity in the mine is achieved using a network of triaxial $15 \mathrm{~Hz}$ geophones and single-component uniaxial accelerometers. In addition to the observed wide distribution of seismicity the dataset also includes an extensive distribution of blasts. Combined, the overall dataset provides a comprehensive ray geometry that can be used to understand the material property variations in the volume of interest. In this study, we restrict our analyses to the use of the blast data. This restricts our inversion to only considering compressional wave variations, but it does eliminate the complications inherent in solving for unknown locations in addition to unknown velocity structure. As discussed earlier, the dataset was subdivided into three, three-month intervals that surround the de-stress blasting of a stope in a sill pillar: the period considered (2015 Q3) is before the blasting of the stope; the next period (2015 Q4) is during the de-stress blasting of this area; and the final period (2016 Q1) is during the extraction and backfill phase for this stope. Each of these datasets (shown in Figure 1) were comprised of roughly 500 blasts, which provides nearly 14,000 ray paths in each period (see Figure 2).
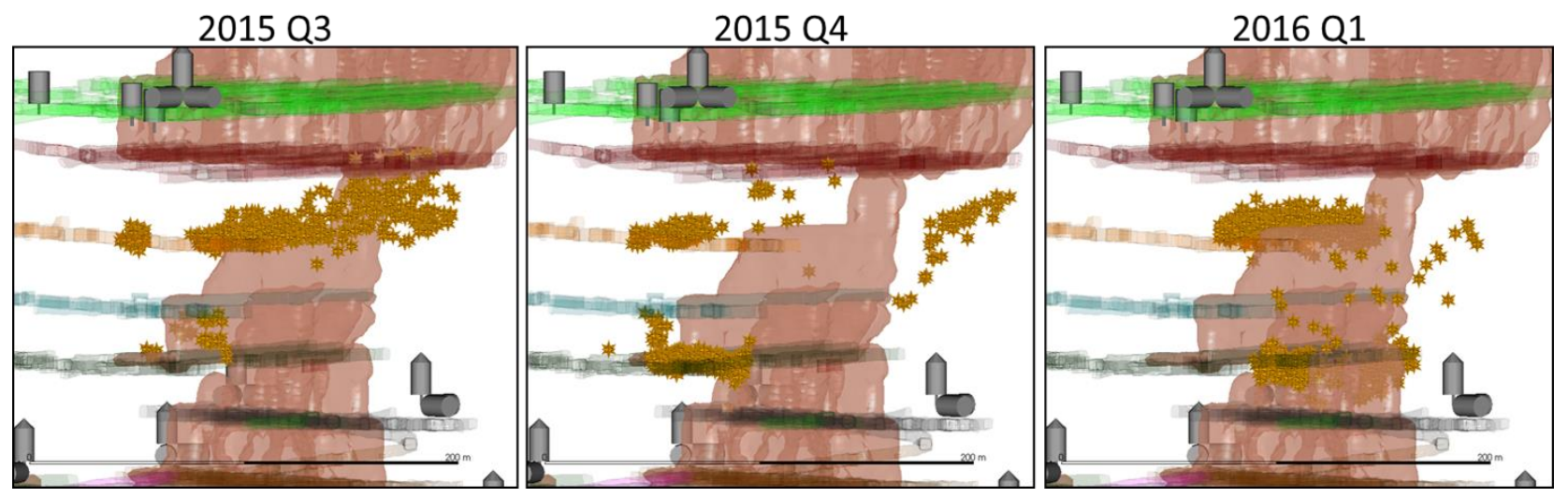

Figure 1 Cross-sectional view of the blasts (stars) used as sources for the tomographic inversions for the three periods considered. The uniaxial sensors are depicted as a single grey cylinder, triaxial sensors as triads of these cylinders, backfilled stopes are shown by their semi-transparent brown outlines, and the other mine workings are coloured by level and are also semi-transparent
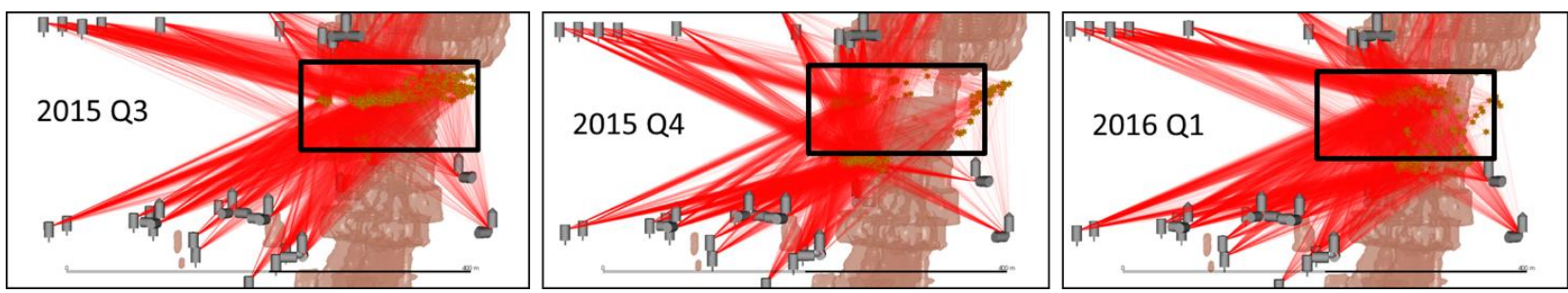

Figure 2 Ray path geometries considered in the inversions in the three quarterly periods (refer to Figure 1 for further details)

\section{3}

\section{Methodology}

\subsection{Tomographic inversion}

We use a tomographic inversion described by Crowley et al. (2015) that is a variation of the simultaneous iterative reconstruction technique, which allows for each inversion to be rapidly evaluated over a series of iterations. This methodology replaces the need to explicitly invert a large system of equations through the use of algebraic reconstruction of the velocities through any cell that a given ray path transects. In other words, we divide the rock mass into blocks, the paths for each ray are evaluated, and the difference in travel time from the predicted, in the simplified background model, are compared to the measured travel times. Each portion of the ray path travel time is divided amongst the cells in the rock mass that intersect the ray paths. 
As mentioned, our current investigation only considers compressional waves from blast data. This simplifies our approach as the locations and timing of the blasts are known and do not have to be solved independently of the velocity inversion. We further simplify the system of equations that we are investigating by not considering complex ray paths that diffract around the backfilled stopes; while we can use a fast marching eikonal algorithm (Rawlinson \& Sambridge 2005) to model the arrival of the first arriving energy, in practice, the complexities of the observed waveforms tends to indicate that these paths may introduce effects that are not well modelled by the ray approximation. Instead, we utilise the simpler ray paths to minimise the introduction of errors into the velocity determinations.

\subsection{Resolution tests}

In any tomographic inversion, it is critical to understand the effects of resolution imposed by the ray geometry on the inverted velocity structure. While it is generally understood that areas that are rich in ray paths crossing the volume at numerous different trajectories result in better resolution relative to areas where ray paths are dominated by a single direction, this assertion is difficult to visualise. In order to show the resolution of the tomographic inversions, we use the relatively standard checkerboard test methodology: a synthetic model of travel time variations is forecast from a velocity structure with alternative blocks of increasing and decreasing velocities. When these synthetic travel times are inverted, areas that recover the input synthetic model are said to be well resolved, and the smearing effects of poorly constraining ray geometry can also be observed in the recovered images. To further test the robustness of the inversions, random picking errors can be added to the input travel times.

In Figure 3, we show a checkerboard test for the ray geometry from the first period in the study in plan view (see Rawlinson \& Sambridge 2003 for a discussion of this methodology). For the image on the left, corresponding to a cut plane at an elevation transecting the top of the stope, a positive and negative 'check' is well recovered, as highlighted by the black square. This good recovery of the initial check pattern suggests that the ray geometry for the blasts and sensors is adequate to recover travel times in this volume. In contrast, if we examine another level in the mine, we see a clear signature of smearing within the checkerboard pattern as outlined by the black oval. Because this area is outside the area of interest, we are less concerned with the ability for the inversion to image velocities here.
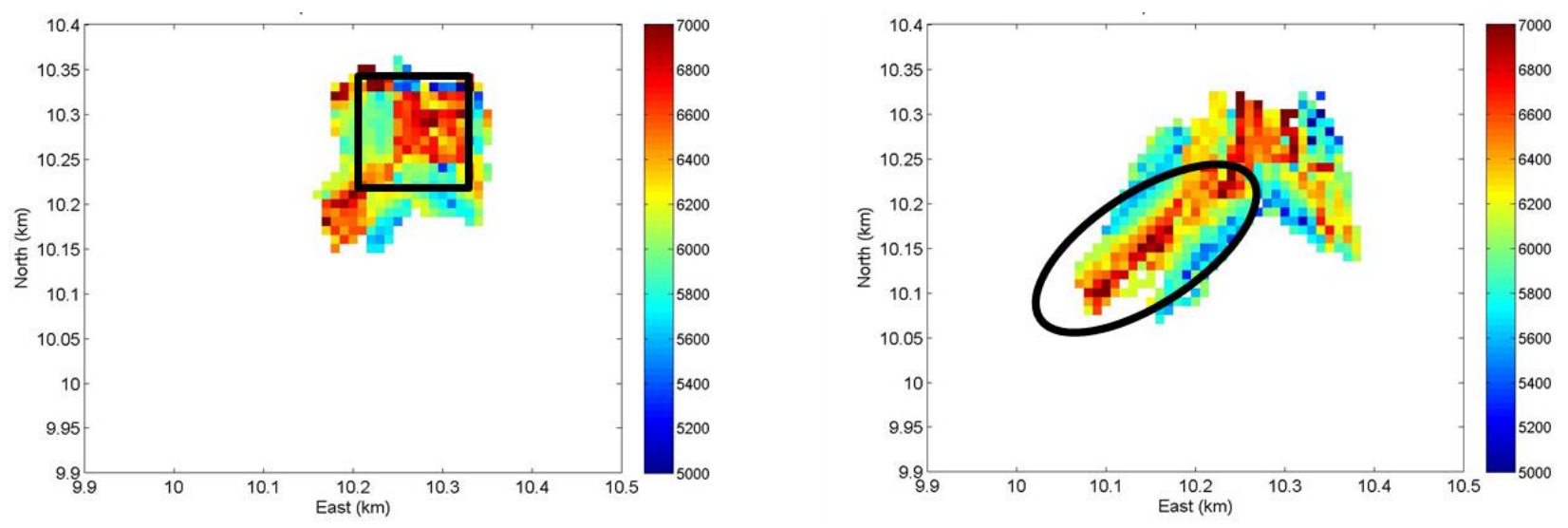

Figure 3 Checkerboard tests at two different levels indicating where potential velocity structure may be well-resolved (on the left, shown by the black square) and where any velocity structure will be smeared (on the right, shown by the black oval)

\section{$4 \quad$ Imaging stress variations}

We use the blast data to image the velocity, and by proxy stress variations in the mine during the nine months of the study. In particular, we look at the three time periods around the de-stress blasting and extraction of the stope in question to attempt to observe the variations in the stress with time. We show the results of the three different inversions in Figure 4. Each column in this figure represents a different three-month interval. The top row shows the different results of the tomographic inversions. Most of the velocity anomalies are 
within $200 \mathrm{~m} / \mathrm{s}$ from the background velocity of the rock mass. In the middle row we show checkerboard tests for the three different array geometries. In general, the areas around the stope are well resolved, but there is some velocity smearing to the southwest in each image. Fortunately, we are not interested in resolving the velocities in this area. Finally, on the bottom row we show how the initial travel time residuals (in red) are decreasing after the inversion (in blue). The median of these distributions is indicated by the coloured vertical line. On average, the variations in the velocity that we image through this methodology (and are taken to be a proxy for stress) are responsible for a factor of three in variation reduction for these travel time residuals.

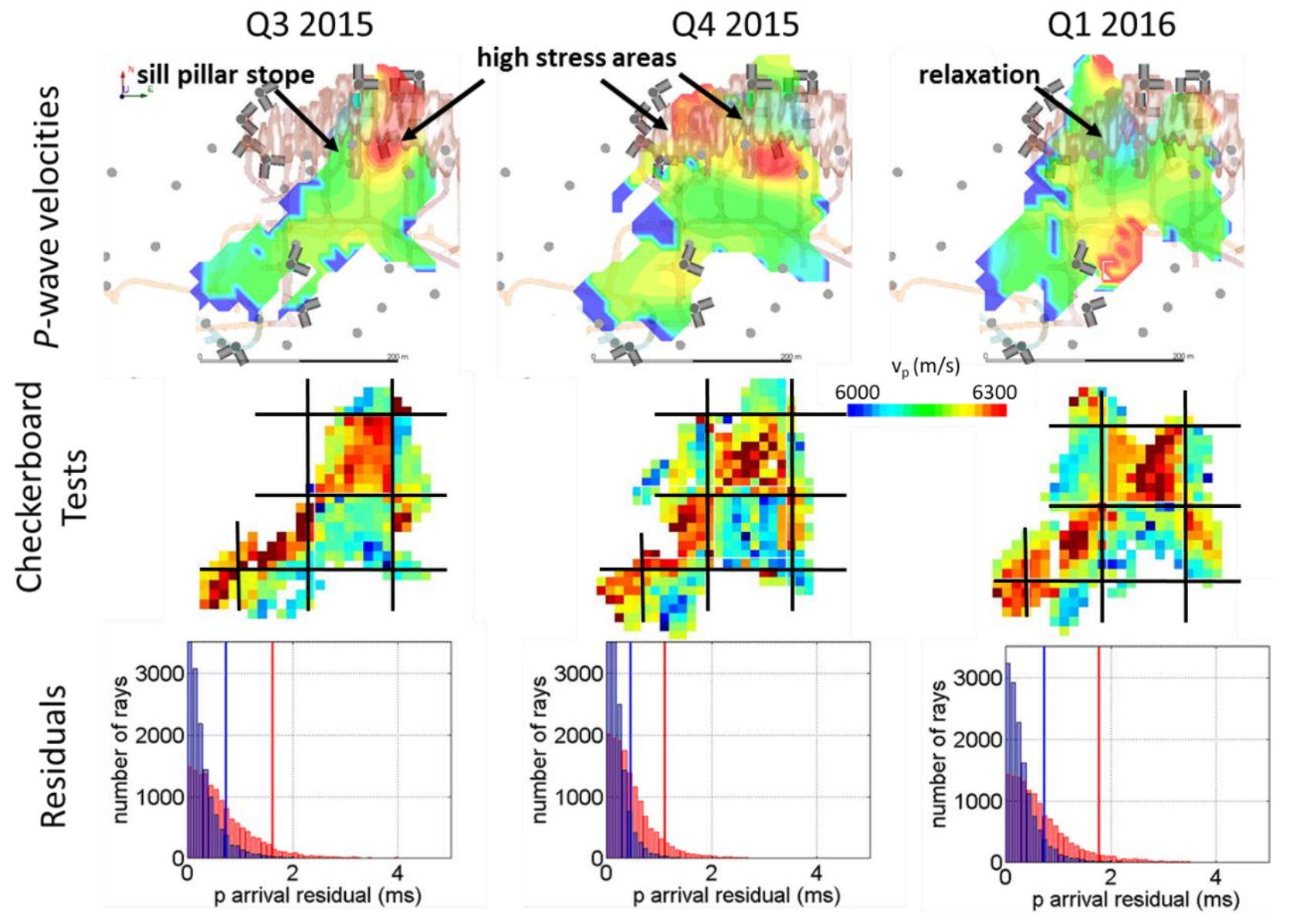

Figure 4 Results of the tomographic inversions (as previously described in detail)

Where we are confident in the recovery of the stress variations, around the stope in the northwest of the images (as indicated from the checkerboard tests) we see a build-up of stress initially in Q3 2015, particularly towards the east. In Q4, we see the high stress area persisting. We are also able to resolve a higher stress area to the west of the stope. Into 2016, the de-stress blasting appears to have been successful as the seismic velocities return to their background values and the high-velocity, high-stress areas appear to have dissipated.

\section{$5 \quad$ Conclusions and perspective}

In this study, we have presented a methodology to understand the time-dependant stress variations within a mine using seismic data recorded over a widely distributed network of geophones and accelerometers. In this embodiment, we focused on using compressional wave information from blasts, which had the advantage of precise locations in space and accurate timing that did not involve having to determine locations and rupture initiation times of microseismicity. The resulting images show a velocity increase in the sill pillar before extraction of the stope, which we interpret as a stress increase. The subsequent time periods, during and after extraction, show that the high velocity region migrates and dissipates after the de-stress blasting 
indicating that stress is being released in the process. This pattern illustrates how tomographic imaging can be used to identify and characterise the dynamic stress field in a mine.

Our continuing focus is on incorporating microseismicity into the inversions. The advantages introduced by utilising events is an increase in the temporal and spatial resolution that can be invoked to understanding the variations in the stress field. In terms of the stress behaviour that we resolved, the stress concentrating in the sill pillar and dissipating after de-stress blasting in the area seems very well resolved. The value of this information will allow for better design of extraction procedures, and potentially allow for input into numerical stress models to build a predictive tool.

\section{Acknowledgement}

We would like to acknowledge the cooperation of an unnamed North American hard rock mine that allowed us to use their data. This paper was aided by the thoughtful review of two anonymous reviewers.

\section{References}

Crowley, JW, Baig, AM \& Urbancic, TI 2015, '4D tomography and deformation from microseismic data', Proceedings of the 85th Annual Meeting of the Society of Exploration Geophysics, New Orleans, Louisiana.

Ma, X, Westman, EC, Fahrman, BP \& Thibodeau, D 2016, 'Imaging of temporal stress redistribution due to triggered seismicity at a deep nickel mine', Geomechanics for Energy and the Environment, vol. 5, pp. 55-64.

Maxwell, S \& Young, R 1996, Seismic imaging of rock mass responses to excavation, Elsevier.

Mercier, J-P, de Beer, W, Mercier, J-P \& Morris, S 2015, 'Evolution of a block cave from time-lapse passive source body-wave traveltime tomography', Geophysics, vol. 80, pp. WA85-WA97.

Rawlinson, N \& Sambridge, M 2003, 'Seismic traveltime tomography of the crust and lithosphere', Advances in Geophysics, vol. 46, pp. 81-198.

Rawlinson, N \& Sambridge, M 2005, 'The fast marching method: an effective tool for tomographic imaging and tracking multiple phases in complex layered media', Exploration Geophysics, vol. 36, pp. 341-350.

Silver, PG, Daley, TM, Niu, F \& Majer, EL 2007, 'Active source monitoring of crosswell seismic travel time for stress-induced changes', Bulletin of the Seismological Society of America, vol. 97, pp. 281-293.

Westman, E, Luxbacher, K \& Schafrik, S 2012, 'Passive seismic tomography for three-dimensional time-lapse imaging of mining-induced rock mass changes', The Leading Edge, vol. 31, pp. 338-345.

Young, R \& Maxwell, S 1992, 'Seismic characterization of a highly stressed rock mass using tomographic imaging and induced seismicity', Journal of Geophysical Research, vol. 97, pp. 12361-12373. 
